\title{
Experimental investigation of internal structure of open-channel flow with intense transport of sediment
}

\author{
Václav Matoušek", Vojtěch Bareš, Jan Krupička, Tomáš Picek, Štěpán Zrostlík \\ Czech Technical University in Prague, Faculty of Civil Engineering, Thákurova 7, 16629 Prague 6, Czech Republic. \\ ${ }^{*}$ Corresponding author. E-mail: v.matousek@fsv.cvut.cz
}

\begin{abstract}
Gravity-driven open-channel flows carrying coarse sediment over an erodible granular deposit are studied. Results of laboratory experiments with artificial sediments in a rectangular tilting flume are described and analyzed. Besides integral quantities such as flow rate of mixture, transport concentration of sediment and hydraulic gradient, the experiments include measurements of the one-dimensional velocity distribution across the flow. A vertical profile of the longitudinal component of local velocity is measured across the vertical axis of symmetry of a flume cross section using three independent measuring methods. Due to strong flow stratification, the velocity profile covers regions of very different local concentrations of sediment from virtually zero concentration to the maximum concentration of bed packing. The layered character of the flow results in a velocity distribution which tends to be different in the transport layer above the bed and in the sediment-free region between the top of the transport layer and the water surface. Velocity profiles and integral flow quantities are analyzed with the aim of evaluating the layered structure of the flow and identifying interfaces in the flow with a developed transport layer above the upper plane bed.
\end{abstract}

Keywords: Acoustic anemometry; Plane bed; Solid-liquid flow; Tilting flume experiment.

\section{INTRODUCTION}

Intense transport of a bed load develops in flow over a mobile bed if the top of the bed is eroded by high applied shear stress. High bed shear is responsible for washing-out of possible bed forms and for the development of a transport layer above the upper-stage plane bed (UPB).

The first laboratory experiment with layered flow at high bed shear was conducted in a pressurized pipe (Wilson, 1966), and so far a majority of experimental data sets have been collected in laboratory pipes (Matoušek, 2009; Matoušek, 2011; Matoušek et al., 2013; Matoušek and Krupička, 2014; Nnadi and Wilson, 1992; Pugh and Wilson, 1999; Sumer et al., 1996). In pressurized pipes, it is easy to establish the steep slope of the energy grade line required to produce the high bed shear condition required at the top of the stationary deposit.

In gravity-driven open-channel flows, the high bed shear stress is produced by the high discharge of sediment-water mixture, or by the steep longitudinal slope of the channel bed, or by the combination of both. In laboratory experiments, the intense-transport condition may be further supported by the use of lightweight sediment (Armanini et al., 2005; Capart and Fraccarollo, 2011; Revil-Baudard et al., 2015).

An analysis of mechanisms governing stratified flows with intense transport of bed load requires information on the internal structure of the flow, i.e. the distribution of velocity and solids concentration across the flow. Experimental results of the internal structure of layered flows in open channels are scarce.

An aim of our investigation is to evaluate the layered structure of the flow and to identify layer interfaces in the flow with a developed transport layer above the upper plane bed using own experiments containing measurements of flow integral quantities and measurements of velocity distribution in the cross sectional vertical of the flow.

\section{LAYERED STRUCTURE OF FLOW}

Our investigations focus on steady uniform, turbulent open channel flows over inclined granular beds in the upper planebed regime. Visual observations of such flows suggest a typical layered structure as shown in Figure 1. Basically, the flow is composed of three layers. Below the water surface, there is a layer of clean water and occasionally saltating particles. Beneath this layer, the transport layer occurs through which the sediment is transported. At the bottom of the layer, i.e. at the top of the deposit (the stationary-bed layer), particles no longer move. From visual observation it seems that the local concentration increases with the vertical position below the top of the transport layer, being effectively zero at the top of the layer and reaching the maximum value at the bottom of the transport layer. Deeper in the transport layer, grains get closer to each other and their contact becomes more frequent. Near the bottom of the transport layer the particles are in virtually permanent contact. The thickness of the individual layers varies considerably with the discharge and/or longitudinal slope of the bed (i.e. inclination angle of the bed).

\section{EXPERIMENTAL WORK \\ Experimental set-up}

Experiments were conducted in the rectangular tilting flume of the Laboratory of Water Engineering of Czech Technical University in Prague, Czech Republic. The recirculating flume can be tilted to steep slopes. It is $0.2-\mathrm{m}$ wide and $8-\mathrm{m}$ long, and additional dimensions are given in Figure 2 (right panel). The system is composed of two parts, one for recirculating sediment-water mixture and the other for recirculating water only (Figure 2, left panel). Both mixture- and water circuits are equipped with a centrifugal pump with a variable frequency drive. 


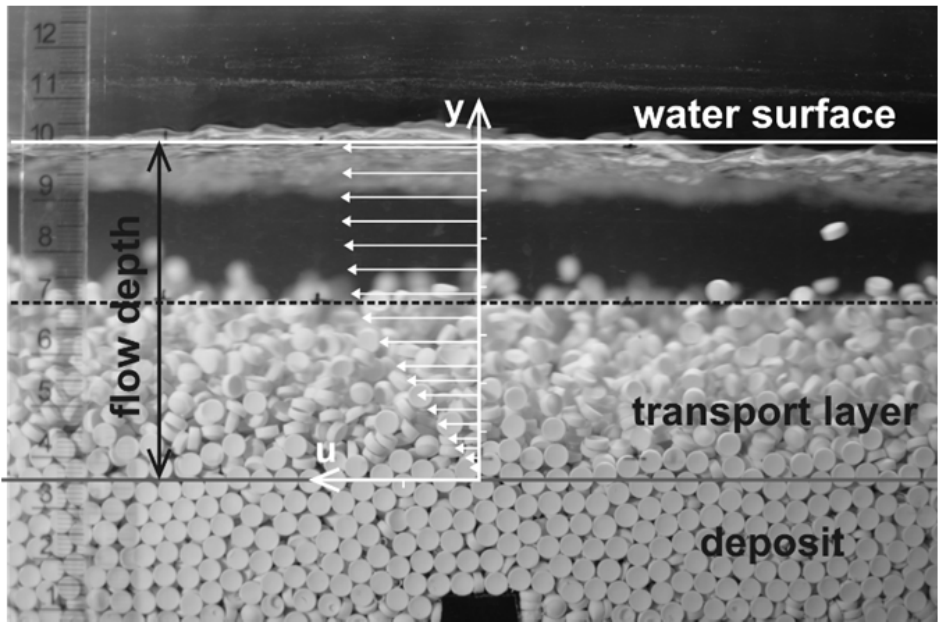

Fig. 1. Schematic layered structure of flow transporting plastic sediment through transport layer above deposit in upper plane-bed regime in flume.

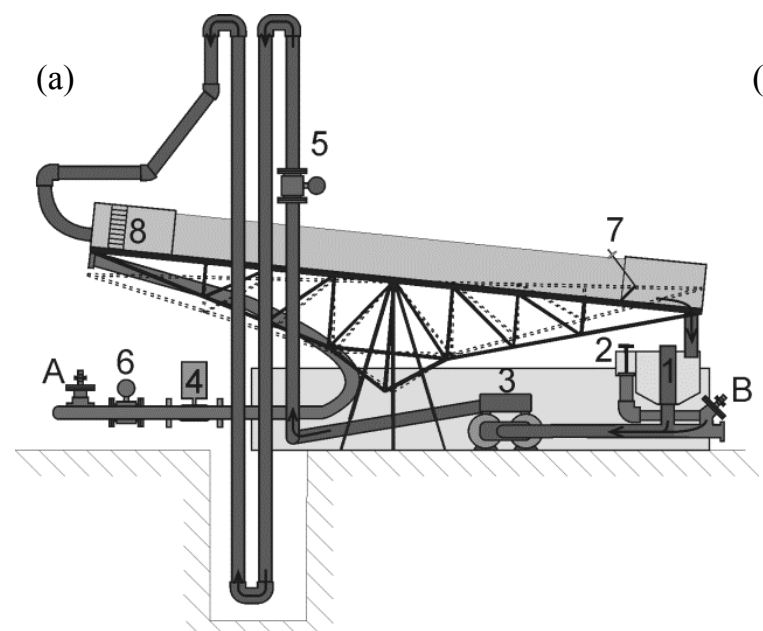

(b)

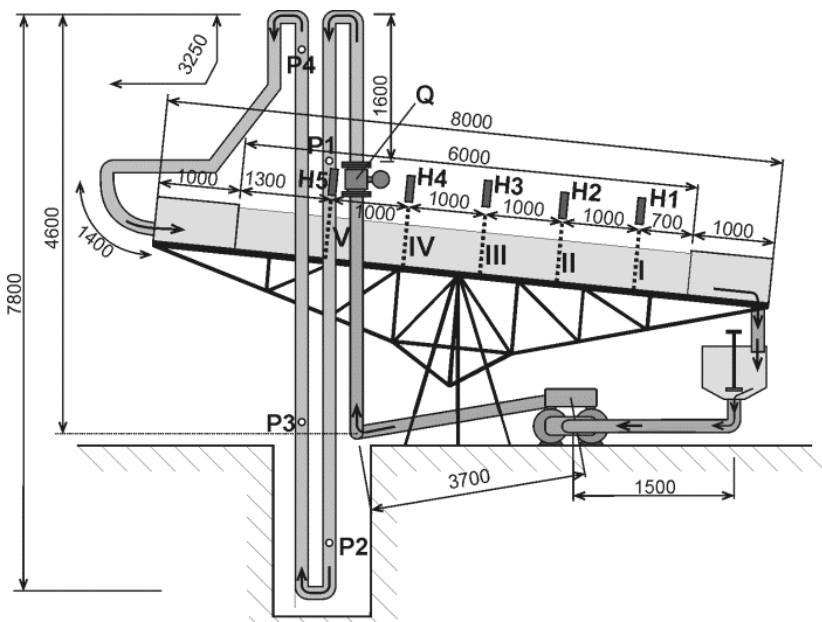

Fig. 2. Layout of experimental set-up for 2015-tests. Legend: (a): 1 - supply and separation tank, 2 - overflow, 3 - centrifugal pump for mixture, 4 - centrifugal pump for water, 5 - flow meter for mixture, 6 - flow meter for water, 7 - outlet with overshot weir, 8 -inlet with flow distributors; A - control valve, $\mathrm{B}$ - inlet valve; (b): $\mathrm{H}$ - ultrasonic gauges for water surface measurement, $\mathrm{P}$ - pressure sensors, $\mathrm{Q}$ flow meter for mixture, distances in $\mathrm{mm}$.

In the connecting pipes of the mixture circuit, the discharge of mixture is measured using the magnetic flow meter (symbol Q in the right panel of Figure 2, KROHNE OPTIFLUX 5000) and the delivered concentration of sediment is determined from the measured pressure differences in the upgoing and downcoming vertical pipes ( $\mathrm{P}$ in the right panel of Figure 2, Rosemount, DP1151). In the flume itself, measurements are carried out in five measuring cross sections (I, II, II, IV, V) during the 2015-tests. The water level is measured using ultrasonic probes $(\mathrm{H}$ in the right panel of Figure 2, PepperlFuchs UC500-30GM-IUR2-V15). The position of the top of the bed and the position of the top of the transport layer are observed visually through the glass side walls of the flume. Furthermore, the inclination angle of the flume is measured.

Three independent methods were used to measure local velocities in the water layer and transport layer above the deposit in the flume. All instruments were located to the same measuring location: to the center of the flow cross section $4.2 \mathrm{~m}$ behind the flume inlet. Each applied method (and instrument) has its limitations and its validity must be evaluated using specific criteria for particular conditions in the tested flows.
The Prandtl tube (PT) senses the local velocity through measuring the differential pressure over its two tubes. The outer diameter of our PT is $6 \mathrm{~mm}$ and the diameter of the frontal hole is $1.0 \mathrm{~mm}$ (Figure 3a). One limitation for using PT is associated with its diameter: the highest measured point can be no higher than $3 \mathrm{~mm}$ below the water surface if the surface does not fluctuate significantly. The other limitation is the sensitivity of the pressure transducer (Siemens SITRANS P range $0.6 \mathrm{~m}$ ) which may affect measurement accuracy of very low velocities.

The Acoustic Doppler Velocity Profiler (ADVP, NORTEK SA Vectrino Profiler) detects a velocity profile in a $32-\mathrm{mm}$ thick part of the flow depth in one measurement. The vertical resolution of the measurement is $1 \mathrm{~mm}$. A major advantage of ADVP is its ability to measure local velocities at different vertical positions simultaneously, its disadvantage is that it is not applicable for shallow flows (Figure 3b). Furthermore, the shape of the probe receivers poses a problem as in the case of small submergence the receivers attract air pockets which gather in the wakes behind receivers and may influence measurements. 


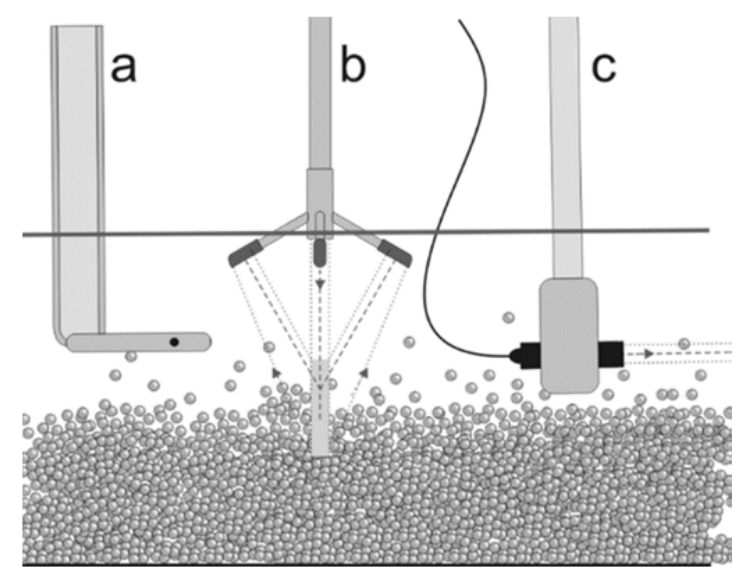

Fig. 3. Schematic details of measuring instruments for velocity profiles. Legend: a - Prandtl tube (PT), b - Acoustic Doppler Velocity Profiler (ADVP), c - Ultrasonic Velocity Profiler (UVP) (Zrostlík et al., 2015).

Table 1. Error estimation for measured and derived quantities.

\begin{tabular}{l|c}
\hline Quantity & $\begin{array}{c}\text { Estimated standard devia- } \\
\text { tion }\end{array}$ \\
\hline \multicolumn{2}{c}{ Measured quantities: } \\
\hline Volumetric discharge & $<0.21 / \mathrm{s}$ \\
\hline Vertical position of bed & $\approx 1.5 \mathrm{~mm}$ \\
\hline Vertical position of water surface & $<0.5 \mathrm{~mm}$ \\
\hline Pressure difference & higher from $2 \%$ and $2 \mathrm{~mm}$ \\
\hline \multicolumn{1}{c}{ Quantities calculated from measured quantities: } \\
\hline Mean velocity & $3 \%$ \\
\hline Depth & $2 \mathrm{~mm}$ \\
\hline Delivered concentration & $1 \%$ of concentration value \\
\hline Longitudinal slope of bed & $2 \mathrm{~mm} / \mathrm{m}$ \\
\hline Shields parameter & $15 \%$ \\
\hline $\begin{array}{l}\text { Difference between local velocity } \\
\text { measured by PT and local veloci- } \\
\text { ty measured by ADVP in flow of } \\
\text { water }\end{array}$ & $5 \mathrm{~mm} / \mathrm{s}$ \\
\hline
\end{tabular}

As with the PT, our Ultrasonic Velocity Profiler (UVP, MetFlow SA) is set to measure only the local longitudinal velocity component of the flow. A $4 \mathrm{MHz}$ transducer is directed against the flow using a support (Figure 3c). The outer diameter of the transducer is $8 \mathrm{~mm}$ and the diameter of the active area is $5 \mathrm{~mm}$. The horizontal distance of the control volume in front of the transducer is $32 \mathrm{~mm}$ in the water layer and $18 \mathrm{~mm}$ in the transport layer. The vertical positions for the measuring points were chosen the same as for PT.
Estimated standard deviations of the measured and derived quantities are given in Table 1 . The resolution of a single velocity measurement by ADVP (or UVP) method given by manufactures is reported as $0.5 \%$ of measured value for ADVP and as $\mathrm{V}_{\max } / 256$ for UVP $\left(\mathrm{V}_{\max }\right.$ is the velocity range). However, the combined measurement uncertainty of mean time-averaged velocity components contains two sources of uncertainty - internal and external uncertainty factors (Furuichi, 2013). Internal factors are related to the pulse repetition frequency, the basic frequency, the time lapse, the raw velocity and sound velocity.

External factors are the sound velocity and incident angle of probes (Furuichi, 2013; Peltier et al., 2013) which can be in many applications the dominant source of errors. Our measurements deal with time-averaged longitudinal components of velocity, where the significance of this source of uncertainty is minimal. Based on the literature review, the sensitivity- and uncertainty analysis, and our sampling strategy (1500 samples for UVP, 5000 samples for ADVP), we conclude that the combined uncertainty is below $1 \%$ of the measured time-averaged velocity component.

More details about the experimental set-up and the measuring techniques can be found in Zrostlík et al. (2015).

\section{Sediment fractions}

In this paper, experimental results are discussed for two tested fractions of plastic sediments (HSF30, TLT25). Both fractions are narrow-graded and of similar size and density (see values of the particle sizes $d_{16}, d_{50}, d_{84}$ from sieving tests and the relative density $S_{s}$ in Table 2; further in Table 2: $w_{t}=$ terminal settling velocity of particle, $C_{v b}=$ loose poured volumetric concentration of particles in bed). However, they differ significantly in grain shape. HSF30-grains are ellipsoidal, while TLT25grains are more rounded, although asymmetrical.

\section{Integral parameters of flow}

Figures 4 and 5 show test results of integral parameters measured for one fraction of sediment (TLT25) and collected over the range of mixture discharges and longitudinal slopes of the bed. The results show that transport of TLT25-sediment is sensitive to the longitudinal slope of the bed. The delivered concentration of transported sediment $C_{v d}$ (defined as the ratio of the volumetric discharge of sediment and the total discharge of mixture) correlates very tightly with the inclination angle of the top of the bed, $\omega$. The bed friction coefficient $\lambda_{b}$ obtained from the measured integral parameters $\left(\lambda_{b}=8 \cdot g \cdot R_{b} \cdot \sin \omega \cdot b^{2} \cdot h^{2} / \mathrm{Q}_{m}^{2}\right.$, where $g=$ acceleration of gravity, $R_{b}=$ side-wall corrected flow depth, i.e. the hydraulic radius of discharge area associated with top of bed, $b=$ rectangular channel width, $h=$ flow depth, $Q_{m}=$ volumetric discharge of mixture) exhibits a relation with the inclination angle as well. Hence, $\lambda_{b}$ and $C_{v d}$ are interrelated and Figure 4 shows that both can multiply its value more than 5 times if $\omega$ increases from 1 degree to 4 degrees in the particular case of the TLT25sediment. The results are consistent with the results obtained for the HSF30-sediment (Matoušek et al., 2014).

Table 2. Properties of tested sediment.

\begin{tabular}{l|l|l|l|l|l|l}
\hline Sediment fraction & $\begin{array}{l}d_{16} \\
{[\mathrm{~mm}]}\end{array}$ & $\begin{array}{l}d_{50} \\
{[\mathrm{~mm}]}\end{array}$ & $\begin{array}{l}d_{84} \\
{[\mathrm{~mm}]}\end{array}$ & $\begin{array}{l}S_{s} \\
{[-]}\end{array}$ & $\begin{array}{l}w_{t} \\
{[\mathrm{~m} / \mathrm{s}]}\end{array}$ & $\begin{array}{l}C_{v b} \\
{[-]}\end{array}$ \\
\hline HSF30 & 3.05 & 3.18 & 3.25 & 1.36 & 0.131 & 0.60 \\
TLT25 & 3.80 & 4.15 & 4.45 & 1.38 & 0.106 & 0.59 \\
\hline
\end{tabular}


(a)

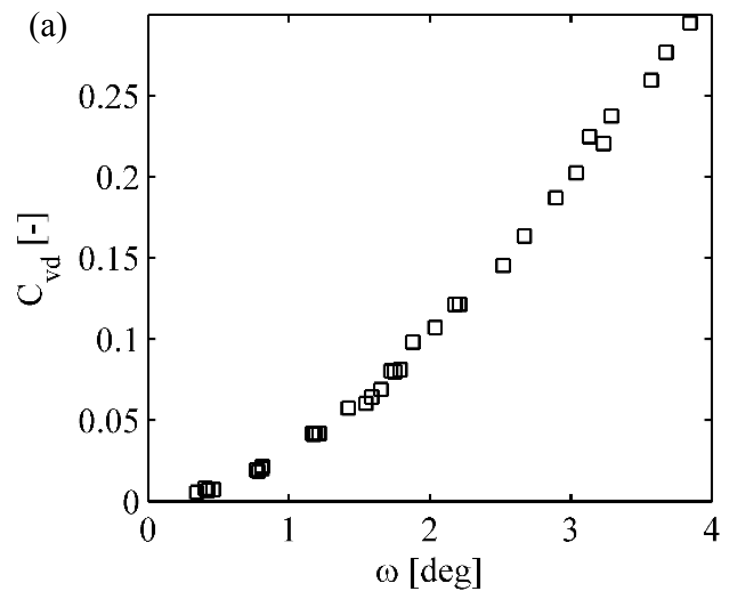

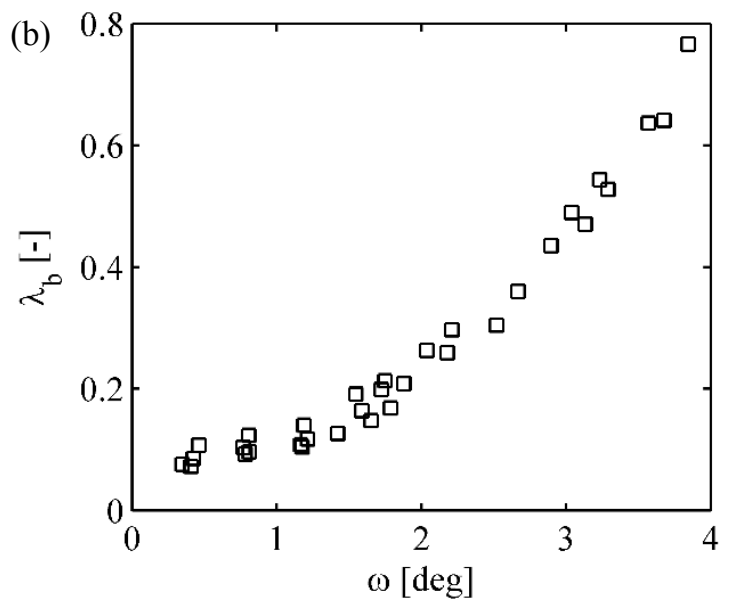

Fig. 4. Variation of delivered concentration of sediment, $C_{v d}$ (a) and bed friction coefficient, $\lambda_{b}$ (b) with inclination angle, $\omega$, of bed for flow with TLT25-sediment.
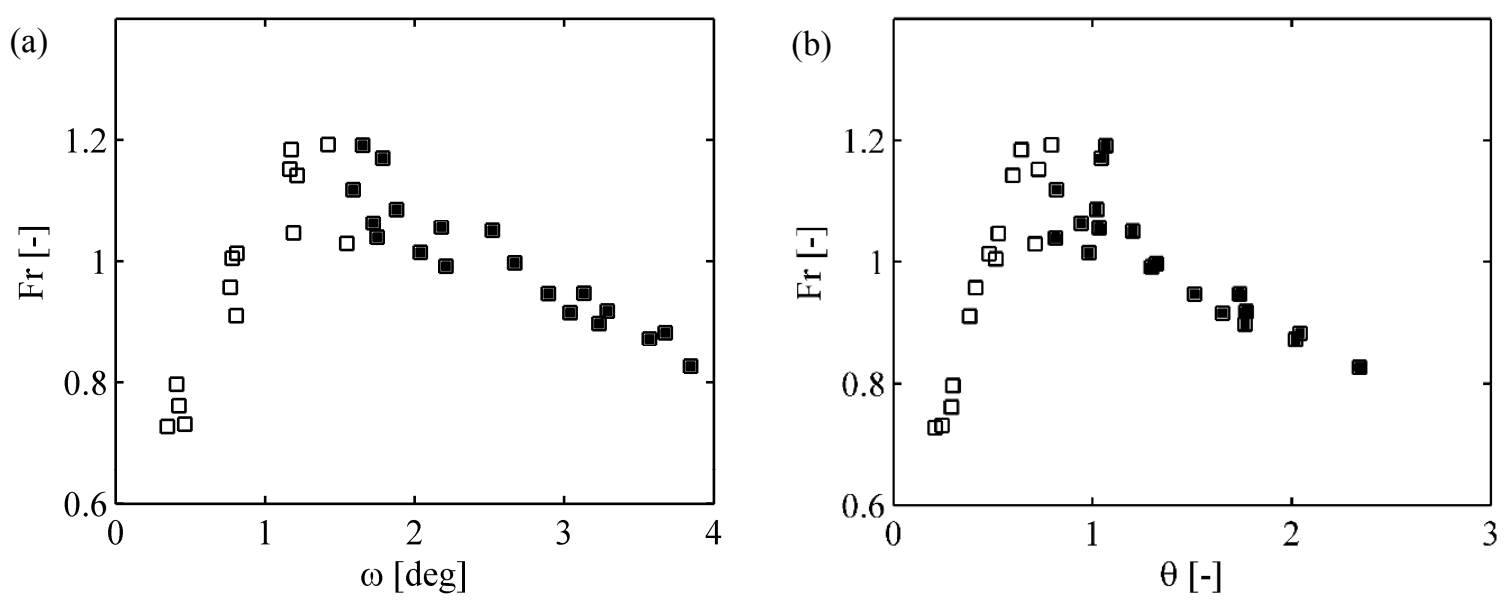

Fig. 5. Variation of Froude number, $F r$, with inclination angle, $\omega$, of bed and with bed Shields parameter $\theta$ for flow with TLT25-sediment. Legend: blank points - flow below threshold value of $\theta$, black points - flow above threshold value of $\theta$.

Figure 5 shows how Froude number $F r=\mathrm{Q}_{m} /\left(g^{0.5} \cdot b \cdot h^{1.5}\right)$ varies with the bed inclination angle and with Shields parameter $\theta=S_{m} \cdot R_{b} \cdot \sin \omega /\left[\left(S_{s}-1\right) \cdot d_{50}\right]\left(S_{m}=\right.$ relative density of mixture). At a certain threshold value of $\theta, F r$ reaches its maximum value (the threshold value $\theta_{t h}$ somewhere between 0.7 and 1.0 in Figure 5). At high values of $C_{v d}$ reached at $\theta$ values higher than the threshold value, $F r$ decreases with the increasing $\theta$ and the trend tends to level-off at the highest values of $\theta$. Also these results are consistent with the results obtained for the HSF30sediment (Matoušek et al., 2014).

\section{Local parameters of flow - velocity distribution}

Measured vertical profiles of the time-averaged longitudinal velocity component, $u$, stretch from the water surface down to the lower part of the transport layer. In Figure 6, the velocities $u$ are plotted against the vertical positions $y(y=0$ at the visually observed top of the deposit) made dimensionless by the flow depth $h$ (left axis) and by the median particle size $d_{50}$ (right axis).

Figure 6 contains plots of two profiles for flow at $\theta<\theta_{t h}$ (a, b) and two profiles for flow at $\theta>\theta_{t h}(\mathrm{c}, \mathrm{d})$. At $\theta<\theta_{t h}$, the water layer (the white area of the plot) dominates over a much thinner transport layer (the grey area). Intense transport of sediment (high $C_{v d}$ at $\theta>\theta_{t h}$ ) is associated with the thicknesses of the transport layer $H_{s h}$ exceeding approximately one half of the total flow depth $h$. Apparently, the shapes of the velocity profiles tend to differ for the transport layer and for the water layer above the transport layer. The profiles may be considered approximately linear in the transport layer. In a case of absent transport layer (the lower plane-bed regime), a velocity profile is logarithmic above the plane bed as confirmed by one of our earlier tests (Zrostlík et al., 2015). A question arises as to whether the log-profile is maintained in the water layer if the transport layer develops (the upper plane-bed regime). 

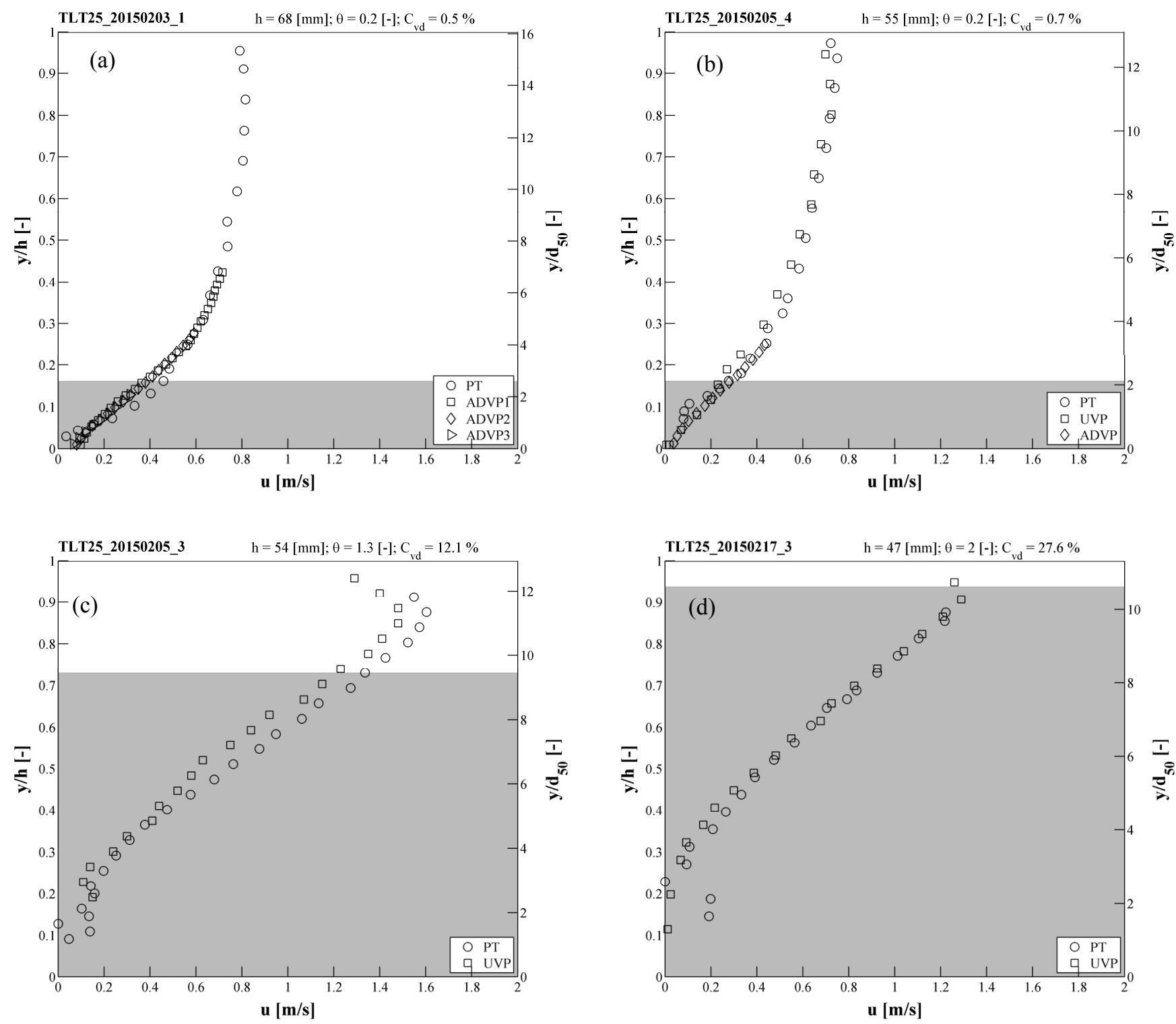

Fig. 6. Velocity profiles measured above and within transport layer using different measuring techniques in flow carrying TLT25-sediment for different test runs (Run Nr. in left upper corner of plots) at different flow depths $h$, Shields parameters $\theta$ and delivered concentrations $C_{v d}$ (values in right upper corner). Legend: points - measured local velocities $u$, grey zone - visually observed transport layer.

\section{EVALUATION OF LAYERED STRUCTURE USING MEASURED VELOCITY PROFILES}

A general formula for the distribution of local velocity, $u$, in the boundary layer above the hydraulically rough boundary predicts a logarithmic velocity profile,

$\frac{u}{u_{* b}}=2.5 \ln \left(B \frac{y-y_{i n i}}{k_{s}}\right)$

in which $u_{* b}=$ shear velocity at the boundary $\left(u_{* b}=\sqrt{g \cdot R_{b} \cdot \sin \omega}\right), \quad B=$ constant (typically $B=30$ ), $y=$ vertical position above the boundary, $y_{i n i}=$ vertical displacement of the profile origin with respect to the position of the flow boundary, and $k_{s}=$ equivalent roughness of the boundary. For layered flows carrying sediments in the UPB regime, we hypothesize that the velocity distribution is logarithmic at least in the part within the water layer above the transport layer provided that the water layer is sufficiently thick compared to the thickness of the transport layer. It is likely that the presence of the transport layer affects $y_{i n i}$ and the other quantities in Equation (1).

In the transport layer itself, the velocity distribution differs from the logarithmic distribution. Based on our results as in Figure 6 and in the earlier publications (Matoušek et al., 2014; Zrostlík et al., 2015) and on observations of others (Armanini et al., 2005; Capart and Fraccarollo, 2011), we assume that the velocity profile is linear below the logarithmic profile,

$\frac{u}{u_{t r}}=\frac{y-\Delta y}{y_{t r}-\Delta y}$

in which $u_{t r}=$ local velocity at the position $y_{t r}$ where the linear profile connects through the same gradient with the logarithmic profile; $\Delta y=$ position of zero velocity (i.e. the displacement of the linear-profile origin with respect to the position of the top of 
the deposit). Moreover, we assume that the velocity profile is linear in a major part of the transport layer,

$\frac{u}{u_{s h}}=\frac{y-\Delta y}{y_{s h}-\Delta y}$

in which $u_{s h}=$ local velocity at the top of the transport layer and $y_{s h}=$ position of the top of the transport layer.

A schematic composition of the velocity profile is shown on the left-hand side of Figure 7. On the right-hand side of Figure 7, the shape of a schematic profile of sediment concentration is given. The concentration profile is considered linear in the transport layer, which is in agreement with earlier experimental findings (e.g. Pugh and Wilson, 1999; Matoušek, 2009).

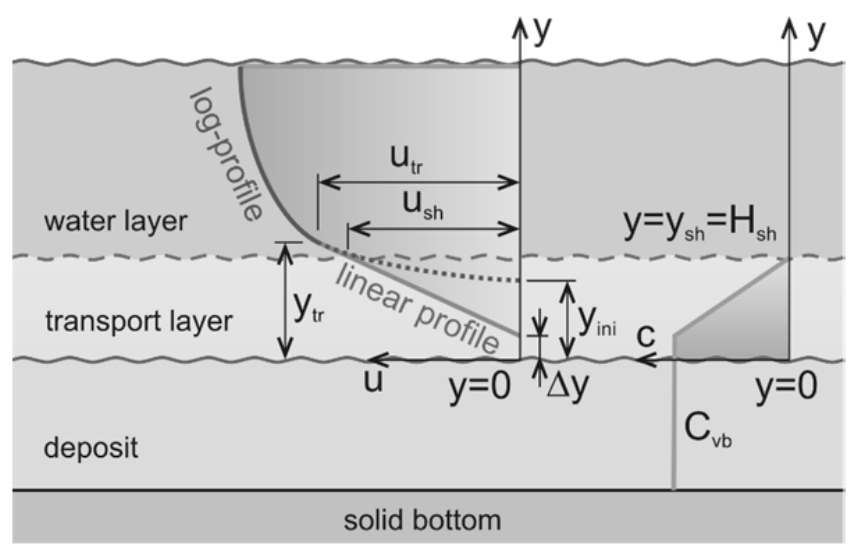

Fig. 7. Schematic structure of flow with composite profile of velocity and linear profile of concentration.

\section{Evaluation of composite profile of longitudinal velocity}

An optimization procedure is employed to produce the best fit (using the least square method) to measured velocity profiles and to find values for selected parameters in Equation (1) and Equation (2a). The evaluation procedure is set to split a measured profile into two parts - the lower part is linear (satisfying Equation 2a) and the upper part is logarithmic (satisfying Equation 1).

Appropriate local velocities from the three measuring methods are selected as reliable input data to the optimization procedure. Five parameters $\left(u_{*_{b}}, k_{s}, y_{i n i}, y_{t r}, \Delta y\right)$ are available for optimization. Results in Figure 8 are reached for $u_{*_{b}}$ values used as inputs (determined from measured integral quantities), i.e. besides $k_{s}$, the characteristic vertical positions in the layered structure $\left(y_{i n i}, y_{t r}, \Delta y\right)$ are the outputs of the optimization procedure.

The interface between the linear part and the logarithmic part of a profile (i.e. the position of $y_{t r}$ ) is indicated by the dotted line in Figure 8. The dashed line indicates the position of the origin of the logarithm profile $\left(y_{i n i}\right)$. In plots a,b,c of Figure 8 , the position of the transition $y_{t r}$ exceeds the position of the visually observed top of the transport layer (the upper range of the grey area), but other observations with both TLT25 (e.g. plot d of Figure 8) and HSF30 revealed that in many cases, $y_{t r} \approx y_{s h}$. In Figure 9, the vertical positions $y_{s h}$ and $y_{t r}$ normalized by the flow depth $h$ are plotted against Shields parameter $\theta$. The plot indicates that the difference between $y_{s h} / h$ and $y_{t r} / h$ is small at each particular $\theta$. Figure 9 also shows that the relative position of the top of the transport layer reaches a maximum at $\theta_{t h}$ and no long- er increases with the further increasing $\theta$. The position of the point of transition for the composite velocity profile exhibits the same trend. Apparently, the threshold value of $\theta$ is associated with the condition at which the transport layer reaches its limit for its expansion towards the water surface.

\section{Interfaces of layered structure of flow}

Results of the optimization procedure applied to the measured velocity profiles helps to identify interfaces additional to the ones identified from a visual observation and shown in Figure 1. The visual observation recognizes the interface between the deposit and the transport layer and the interface between the transport layer and the water layer. A determination of the positions of these interfaces is associated with some uncertainty as they tend to fluctuate in time.

The processed velocity profiles indicate an additional interface which is given by the position of the transition at the composite velocity profile, $y_{t r}$. Moreover, the existence of the vertical displacement of the origin of the linear profile $\Delta y$ suggests that another interface needs to be identified within the transport layer at the position $\Delta y$. This is in agreement with some works in literature (Armanini et al., 2005; Capart and Fraccarollo, 2011; Revil-Baudard et al., 2015). Our visual observations reveal that the position of $\Delta y$ corresponds with the position at which collisional interactions typical for the region with the linear velocity profile change to virtually permanent contacts among grains sliding over each other. This sub-layer of sliding grains is negligible at low $\theta$ but tends to grow to almost $40 \%$ of the transport-layer thickness (i.e. 4 to 5 grain layers) at the highest values of $\theta$ for HSF30-tests (Zrostlík et al., 2015). A very similar result has been obtained for TLT25 (e.g. Fig. 8d).

Measured velocity profiles exhibit the same trends in a development of the layered structure of the flow for both plastic fractions tested. The threshold value of $\theta$ plays an important role in describing the trends. To examine the trends in a quantitative way, we determine values of the Richardson number

$$
R i=\frac{-g \cos \omega\left(S_{s}-1\right)(d c / d y)}{(d u / d y)^{2}}
$$

in which $\mathrm{d} c / \mathrm{d} y=$ concentration gradient, and $\mathrm{d} u / \mathrm{d} y=$ velocity gradient. For the linear (collisional) part of the transport layer, the concentration gradient is $-C_{v b} /\left(y_{s h}-\Delta y\right)$ and the velocity gradient equals to $u_{s h} /\left(y_{s h}-\Delta y\right)$ so that Equation (3) simplifies to

$R i_{s h}=\frac{\left(S_{s}-1\right) C_{v b}\left(y_{s h}-\Delta y\right) g \cos \omega}{u_{s h}^{2}}$

for the Richardson number of the collisional part of the transport layer. The results in Figure 10 show that the collisional $R i_{s h}$ stops decreasing at $\theta_{t h}$ and remains constant $\left(R i_{s h} \approx 0.035\right)$ at $\theta>\theta_{t h}\left(\theta_{t h} \approx 1.1\right.$ for HSF30, a slightly lower value for TLT25).

In Figure 10 , the $R i_{s h}$-values are obtained for $C_{v b}=0.6$. Equation 4 contains two other parameters associated with the collisional part of the transport layer: the thickness of the collisional part of the transport layer, $y_{s h}-\Delta y$, and the local velocity $u_{s h}$. Figures 11 and 12 show that they are both very sensitive to $\theta$ at $\theta<\theta_{t h}$, and virtually insensitive to $\theta$ at $\theta>\theta_{t h}$. 

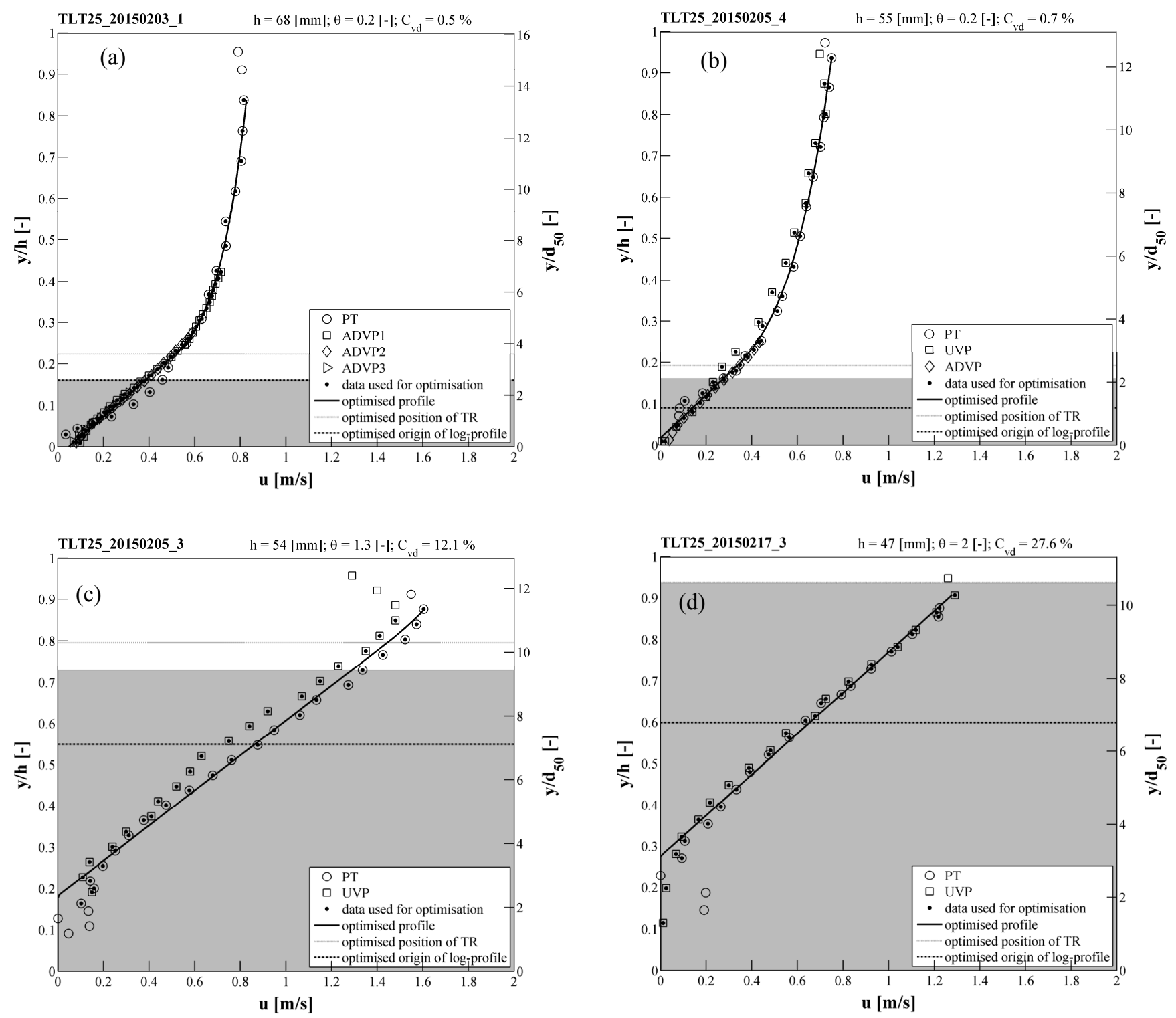

Fig. 8. Measured velocity profiles evaluated by optimization procedure for composite profile of velocity (TL25T-sediment). Legend: thick line - optimized composite velocity profile, grey line - position of $y_{t r}$, dashed line $=$ position of $y_{i n i}$.

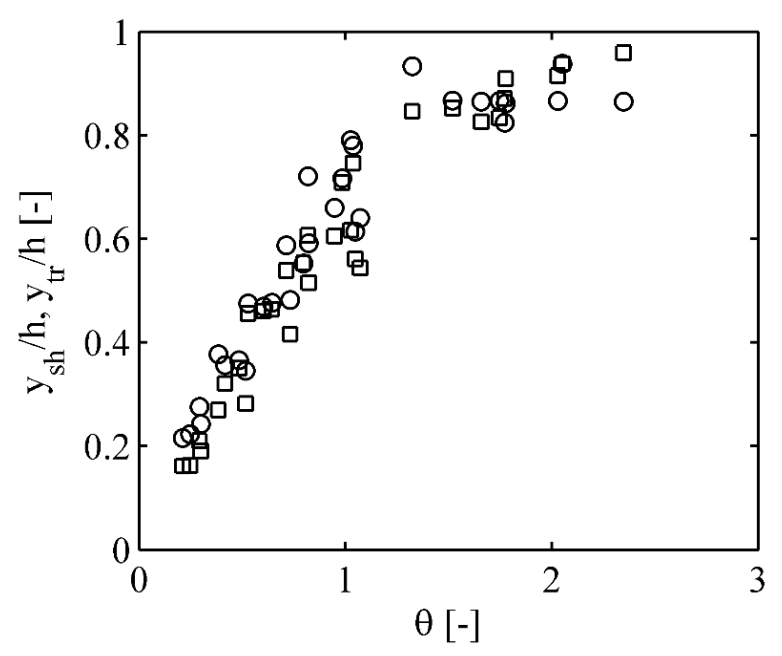

Fig. 9. Relative vertical positions of interfaces versus bed Shields parameter for TLT25. Legend: squares $-\mathrm{y}_{\mathrm{sh}} / \mathrm{h}$, circles $-\mathrm{y}_{\mathrm{tr}} / \mathrm{h}$.
Based on these results, it can be hypothesized that a collisional layer of an almost constant thickness and constant gradients of velocity and concentration develops in the upper part of the transport layer at very high bed shear $\left(\theta>\theta_{t h}\right)$. Although the flow depth $h$ and the thickness of the entire transport layer $H_{s h}$ tend to increase with the increasing $\theta$ at $\theta>\theta_{\text {th }}$, the collisional layer remains unchanged and the increase of the total depth and the transport layer is due primarily to the thickening of the sliding granular sub-layer below the collisional part of the transport layer.

In flows at such high bed shear, the transport layer accounts for a major part of the flow depth and a development of its structure seems to be governed by the 'self-adjusting' mechanism that keeps gravity-driven stratified shear flows in an equilibrium state characterized by a constant value of Richardson number as suggested by Capart and Fraccarollo (2011). A development of the sliding sub-layer at the bottom of the transport layer enables a continuing increase of the mean concentration of transported sediment $C_{v d}$ (and $h$ and $H_{s h}$ ) with the increasing $\theta$ at $\theta>\theta_{t h}$, where the thickness of the collisional 

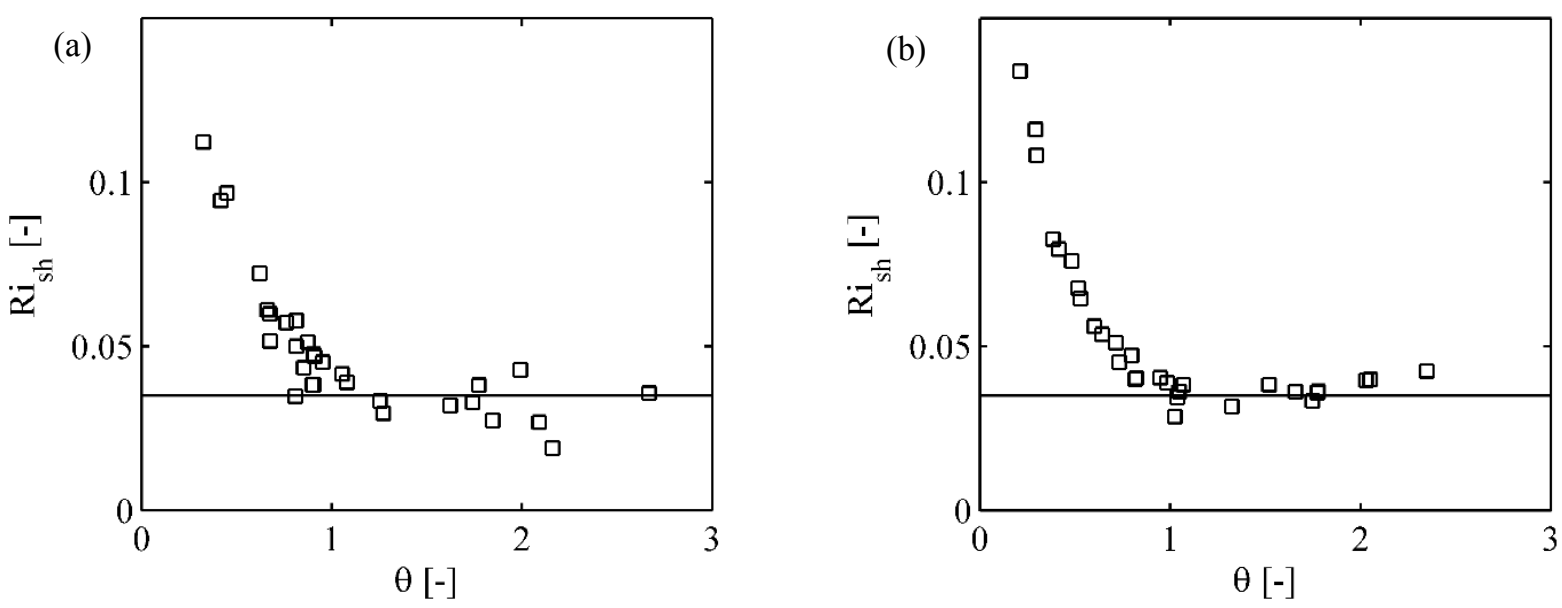

Fig. 10. Richardson number for linear part of transport layer related to bed Shields parameter; (a) for HSF30; (b) for TLT25. Legend: squares - experimental results, line $-R i_{s h}=0.035$

part of the transport layer no longer grows. The observations need to be verified by more tests and analyzes which is work currently in progress.

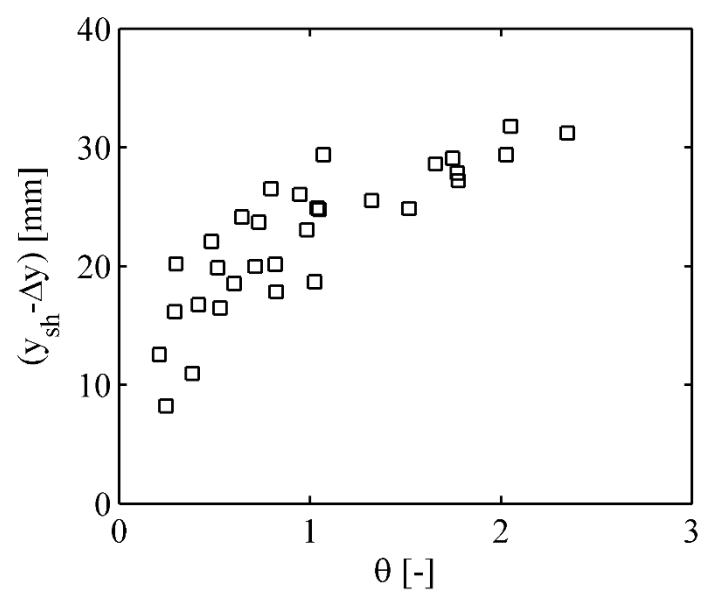

Fig. 11. Thickness of linear-profile collisional layer related to bed Shields parameter for TLT25.

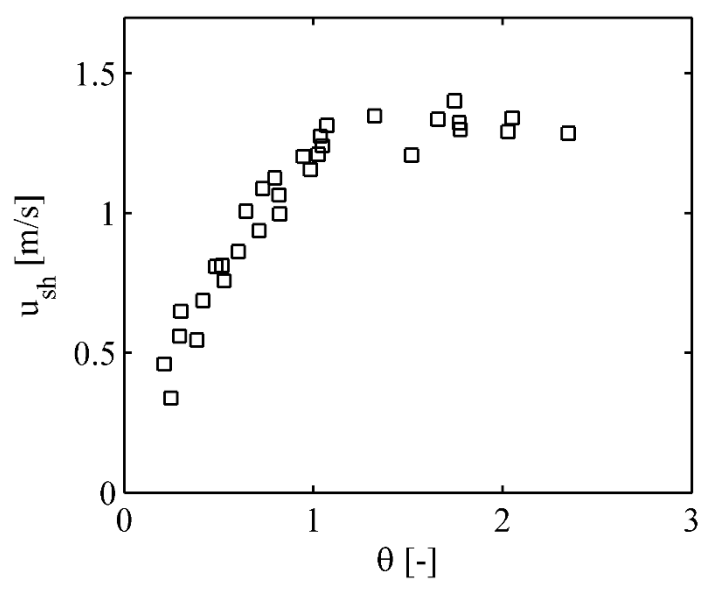

Fig. 12. Local velocity at top of transport layer related to bed Shields parameter for TLT25.

\section{CONCLUSIONS}

Measurements of vertical distribution of longitudinal velocity help to identify interfaces in the gravity-driven stratified flows of plastic sediments at high bed shear. A hybrid velocity profile develops in the stratified flow and it is composed of a logarithmic part and a linear part. The logarithmic part spans over a grain-free layer and the linear part over a collisional transport layer. The interface is approximately at the top of the transport layer.

At weak-transport condition, the logarithmic part occupies a bigger portion of the total depth than the linear part. The vertical displacement of the linear-profile origin above the top of the bed is virtually negligible and never exceeds two particle sizes.

At intense-transport condition, the transport layer occupies a major part of the flow depth. Its collisional part with a linear profile of the origin shifted above the top of the bed reaches the constant thickness and velocity gradient. A flow condition is identified at which the collisional layer starts to dominate the internal structure of the flow. The condition is associated with a definite value of the collisional-layer Richardson number reached at a certain threshold value of the bed Shields parameter.

Acknowledgement. The research is funded by the Czech Science Foundation through the grant project No. P105/12/1082.

\section{REFERENCES}

Armanini, A., Capart, H., Fraccarollo, L., Larcher, M., 2005. Rheological stratification in experimental free-surface flows of granular-liquid mixtures. Journal of Fluid Mechanics, 532, 269-319.

Capart, H., Fraccarollo, L., 2011. Transport layer structure in intense bed-load. Geophysical Research Letters, 38, L20402.

Furuichi, N., 2013. Fundamental uncertainty analysis of flow rate measurement using the ultrasonic Doppler velocity profile method. Flow Measurement and Instrumentation, 33, 202-211.

Matoušek, V., 2009. Concentration profiles and solids transport above stationary deposit in enclosed conduit. Journal of Hydraulic Engineering ASCE, 135, 12, 1101-1106. 
Matoušek, V., 2011. Solids transport formula in predictive model for pipe flow of slurry above deposit. Particulate Science and Technology, 29, 1, 89-106.

Matoušek, V., Krupička, J., 2014. Interfacial friction and transport in stratified flows. In: Proceedings of the ICE Maritime Engineering, 167, 3, 125-134.

Matoušek, V., Krupička, J., Picek, T., 2013. Validation of transport and friction formulae for upper plane bed by experiments in rectangular pipes. Journal of Hydrology and Hydromechanics, 61, 2, 120-125.

Matoušek, V., Bareš, V., Krupička, J., Picek, T., Zrostlík, Š., 2014. Experimental evaluation of interfacial friction and transport in steep flume. In: Proc. Int. Conf. Hydrotransport 19, BHR Group, Golden, Colorado.

Nnadi, F.N., Wilson, K.C., 1992. Motion of contact-load particles at high shear stress. Journal of Hydraulic Engineering, $118,12,1670-1684$.

Peltier, Y., Riviere, N., Proust, S., Mignot, E., Paquier, A., Shiono, K., 2013. Estimation of the error on the mean velocity and on the Reynolds stress due to a misoriented ADV probe in the horizontal plane: Case of experiments in a compound open-channel. Flow Measurement and Instrumentation, 34, 34-41.

Pugh, F.J., Wilson, K.C. 1999. Velocity and concentration distributions in sheet flow above plane beds. Journal of Hydraulic Engineering, 125, 2, 117-125.

Revil-Baudard, T., Chauchat, J., Hurther, D., Barraud, P.A., 2015. Investigation of sheet-flow processes based on novel acoustic high-resolution velocity and concentration measurements. Journal of Fluid Mechanics, 767, 1-30.

Sumer, B.M., Kozakiewicz, A., Fredsøe, J., Deigaard, R., 1996. Velocity and concentration profiles in sheet-flow layer of movable bed. Journal of Hydraulic Engineering, 122, 10, 549-558.

Wilson, K.C., 1966. Bed-load transport at high shear stress. Journal of Hydraulic Division ASCE, 92, 6, 49-59.

Zrostlík, Š., Bareš, V., Krupička, J., Picek, T., Matoušek, V., 2015. One-dimensional velocity profiles in open-channel flow with intense transport of coarse sediment. In: EPJ Web of Conferences, 92, 02120. DOI: 10.1051/epjconf/20159202120.

\section{NOMENCLATURE}

$b[\mathrm{~m}]=$ width of rectangular channel

$B[-]=$ constant in equation for velocity distribution above smooth boundary

$c[-]=$ local volumetric concentration of particles

$C_{v b}[-]=$ loose poured volumetric concentration of particles in bed

$C_{v d}[-]=$ delivered volumetric concentration of particles

$d_{16}[\mathrm{~m}]=$ size fraction of sediment sample below which 16 percent of sample is smaller

$d_{50}[\mathrm{~m}]=$ size fraction of sediment sample below which 50 percent of sample is smaller (median particle size)

$d_{84}[\mathrm{~m}]=$ size fraction of sediment sample below which 84 percent of sample is smaller

$d c / d y\left[\mathrm{~m}^{-1}\right]=$ gradient of local volumetric concentration

$d u / d y\left[\mathrm{~s}^{-1}\right]=$ gradient of local longitudinal velocity

$\operatorname{Fr}[-]=$ Froude number

$g\left[\mathrm{~m} / \mathrm{s}^{2}\right]=$ acceleration due to gravity

$h[\mathrm{~m}]=$ flow depth

$H_{s h}[\mathrm{~m}]=$ thickness of the transport layer

$k_{s}[\mathrm{~m}]=$ equivalent roughness of the boundary

$Q_{m}\left[\mathrm{~m}^{3} / \mathrm{s}\right]=$ volumetric discharge of sediment-water mixture

$R_{b}[\mathrm{~m}]=$ hydraulic radius of discharge area associated with bed $=$ flow depth after side-wall correction

$R i[-]=$ Richardson number

$R i_{s h}[-]=$ Richardson number of the collisional part of the transport layer

$S_{m}[-]=$ relative density of sediment-water mixture

$S_{s}[-]=$ relative density of particles

$u[\mathrm{~m} / \mathrm{s}]=$ time-averaged local longitudinal velocity

$u_{s h}[\mathrm{~m} / \mathrm{s}]=$ time-averaged local velocity at the top of the transport layer

$u_{t r}[\mathrm{~m} / \mathrm{s}]=$ time-averaged local velocity at the position $y_{t r}$

$u_{*_{b}}[\mathrm{~m} / \mathrm{s}]=$ bed shear velocity

$w_{t}[\mathrm{~m} / \mathrm{s}]=$ terminal settling velocity of grain

$y[\mathrm{~m}]=$ vertical position above the top of the bed

$y_{i n i}[\mathrm{~m}]=$ vertical displacement of the logarithmic profile origin

$y_{s h}[\mathrm{~m}]=$ position of the top of the transport layer

$y_{t r}[\mathrm{~m}]=$ position of transition at composite velocity profile

$\Delta y[\mathrm{~m}]=$ vertical displacement of the linear profile origin

$\theta[-]=$ bed Shields parameter

$\theta_{t h}[-]=$ threshold value of bed Shields parameter

$\lambda_{b}[-]=$ bed friction coefficient

$\omega[\mathrm{deg}]=$ inclination angle of the top of the bed

Received 12 June 2015

Accepted 4 July 2015 